\title{
Nonlocal Symmetries and Generation of Solutions for Partial Differential Equations
}

\author{
Valentyn TYCHYNIN ${ }^{\dagger}$, Olga PETROVA $\ddagger$ and Olesya TERTYSHNYK ${ }^{\ddagger}$ \\ $\dagger$ Prydniprovs'ka State Academy of Civil Engineering and Architecture, \\ 24a Chernyshevsky Str., Dnipropetrovsk, 49005 Ukraine \\ E-mail:tychynin@mail.pgasa.dp.ua,tychynin@ukr.net \\ $\ddagger$ Dnipropetrovsk National University, 13 Naukovyi Per., Dnipropetrovsk, 49050 Ukraine
}

Received January 06, 2006, in final form January 17, 2007; Published online February 06, 2007

Original article is available at http://www.emis.de/journals/SIGMA/2007/019/

\begin{abstract}
We have constructed new formulae for generation of solutions for the nonlinear heat equation and for the Burgers equation that are based on linearizing nonlocal transformations and on nonlocal symmetries of linear equations. Found nonlocal symmetries and formulae of nonlocal nonlinear superposition of solutions of these equations were used then for construction of chains of exact solutions. Linearization by means of the Legendre transformations of a second-order PDE with three independent variables allowed to obtain nonlocal superposition formulae for solutions of this equation, and to generate new solutions from group invariant solutions of a linear equation.
\end{abstract}

Key words: Lie classical symmetry; nonlocal symmetries; formulae for generation of solutions; nonlinear superposition principle

2000 Mathematics Subject Classification: 35A30; 35K55; 35K57; 35L70

\section{Introduction}

Nonlocal symmetries of nonlinear equations of mathematical physics stay in the center of attention of many authors $[1,2,3,4,5]$. Methods used for investigation of differential equations (DEs) include application of a wide spectrum of nonclassical and nonlocal symmetries of DEs $[6,7,8,9,10,11]$.

Knowing the symmetries we can construct exact solutions for the equations and then proceed with their generating, as well as describe sets of conserved quantities, reduce the initial equation to the equations with smaller number of variables and solve other problems related to these equations.

The method of studying of the group invariance properties of the DEs, created by S. Lie, has been further developed by G. Birkhoff, L.V. Ovsyannikov, P.J. Olver, W.I. Fushchych, N.H. Ibragimov, G. Bluman, J.D. Cole, P. Winternitz and many others.

In the 1970s V.A. Fok [16] found the symmetry of hydrogen atom in Coulomb field, which, as became clear later, could not be found by the Lie method. Then other similar facts started to arise, and it required an adequate explanation. Resumption of interest to Bäcklund transformations [17] in the 1950s and discovery of the Miura transformation allowed explaining of existence of an infinite set of conservation laws for Korteweg-de Vries equation, and active period of finding of further generalizations of S. Lie theory had started.

One of such generalizations, conditional symmetries of DEs [23], that are referred the literature also as nonclassical or weak symmetries [6, 7, 12], may be found by adding of some condition to the equation with this conditional symmetry being the symmetry of the resulting system. Usually this condition is an equation of a surface invariant under infinitesimal operator admitted by the equation under investigation [13, 14]. 
Theory of Lie-Bäcklund group transformations was developed by R.L. Anderson and N.H. Ibragimov in the 1970s [5, 10, 11, 15]. Such transformations forming a one-parametrical group depend on derivatives

$$
\tilde{x}_{i}=f^{i}(x, u, \underset{1}{u}, \ldots, \underset{k}{u} ; \varepsilon), \quad \tilde{u}^{a}=g^{a}(x, u, \underset{1}{u}, \ldots, \underset{k}{u} ; \varepsilon) .
$$

In these formulae and elsewhere $x=\left(x_{0}, x_{1}, \ldots, x_{n}\right)$ is a set of independent variables, and $u=\left(u^{1}, u^{2}, \ldots, u^{m}\right)$ is a set of dependent variables. The corresponding infinitesimal operator has the following form:

$$
X=\xi^{i}(x, u, \underset{1}{u}, \ldots, \underset{k}{u}) \partial_{i}+\eta^{a}(x, u, \underset{1}{u}, \ldots, \underset{k}{u}) \partial_{u^{a}}, \quad i=0,1, \ldots, n, \quad a=1,2, \ldots, m .
$$

Lie-Bäcklund symmetries defined in such a way present the same concept as generalized vector fields in P.J. Olver's terminology [18]. Dependence of expressions in (1) on derivatives is a reason for regarding such symmetry as nonlocal. Note that reversion of the transformation (1) requires integration procedure.

Further in the paper we use the following standard notations:

$$
\begin{aligned}
& u_{\mu}=\frac{\partial u}{\partial x^{\mu}} \equiv \partial_{\mu} u, \quad \mu=0,1,2, \ldots, n-1, \quad \underset{1}{u}=\left\{u_{\mu}\right\}, \\
& u_{\mu \nu}=\frac{\partial^{2} u}{\partial x^{\mu} \partial x^{\nu}} \equiv \partial_{\mu} \partial_{\nu} u, \quad \mu, \nu=0,1,2, \ldots, n-1, \quad \underset{2}{u}=\left\{u_{\mu \nu}\right\} .
\end{aligned}
$$

Another direction in investigation of nonlocal symmetries is based on representation of the equation

$$
F_{1}(x, u, \underset{1}{u}, \ldots, \underset{k}{u})=0
$$

in the form of a conservation law

$$
F_{i}(x, u, \underset{1}{u}, \ldots, \underset{k}{u})=D_{1} \phi^{1}(x, u, \underset{1}{u}, \ldots, \underset{r}{u})+D_{2} \phi^{2}(x, u, \underset{1}{u}, \ldots, \underset{r}{u})=0
$$

and on introduction of a new potential variable by the relation

$$
D_{2} v=\phi^{1}(x, u, \underset{1}{u}, \ldots, \underset{r}{u}) .
$$

It allows studying of a "potential symmetry" of the equation as a classical symmetry of system $[1,3]$

$$
F_{i}(x, u, \underset{1}{u}, \ldots, \underset{k}{u})=0, \quad D_{2} v=\phi^{1}(x, u, \underset{1}{u}, \ldots, \underset{r}{u}), \quad D_{1} v=\phi^{2}(x, u, u, \ldots, u) .
$$

It may be noted that in the described method for investigation of nonlocal symmetries of DE an important place belongs to the recursion operator for an equation introduced by P.J. Olver [18].

Further development of this approach was obtained in [19, 20] via reversion of the recursion operator. Nonlocal symmetries of the pseudopotential type in sense of prolongation structures of Estabrook and Wahlquist were considered in [21]. It was discovered in the paper [22] that the Jacobi identity for characteristics of nonlocal vector fields "appears to fail for the usual characteristic computations" that led to the notion called "ghost symmetries". That also showed impossibility of discussion of Lie algebraic properties for general nonlocal symmetries of DEs.

Utilization of the method of external differential forms by E. Cartan allowed K. Harrison and C.J. Papachristou [4] to calculate classical point (internal or geometrical) symmetry by means of so-called isovectors generated by a vector field

$$
V=\xi^{i}(x, u) \partial_{i}+\eta^{a}(x, u) \partial_{u^{a}} .
$$


So the internal symmetry of DE was determined by the requirement of invariance of an ideal of external differential forms of the system $\gamma_{i}$ that was generated by the equation under action of the Lie derivative $£_{V}$ with respect to vector field $V$

$$
£_{V} \gamma_{i}=b_{i}{ }^{k} \gamma_{k}+\Lambda_{i}{ }^{k} \gamma_{k}+\gamma_{k} M_{i}^{k} .
$$

Nonlocal symmetry of the given equation was studied in [4] with dependence of factors $V$ on derivatives included into this scheme.

Geometrical theory of nonlocal symmetries of DEs has been developed by A.M. Vinogradov and I.S. Krasil'shchik [9] and formulated in the language of jet fiber bundles for the functions being solutions of the given equation.

In this paper we continue investigation of nonlocal symmetries of PDEs [34, 24] by means of nonlocal transformations of variables

$$
\begin{aligned}
& B^{p}(x, u, \underset{1}{u}, \ldots, \underset{k}{u} ; y, v \underset{1}{v}, \ldots, \underset{t}{v})=0, \\
& p=1,2, \ldots, n+m, \quad u=\left\{u^{a}\right\}, \quad a=1,2, \ldots, m
\end{aligned}
$$

or, in a simpler form

$$
\tau:\left\{\begin{array}{l}
x_{i}=h^{i}(y, v, \underset{1}{v}, \ldots, \underset{k}{v}), \\
u^{a}=H^{a}(y, v, \underset{1}{v}, \ldots, \underset{k}{v}) .
\end{array}\right.
$$

Under the transformation $\tau$ the equation

$$
F_{1}(x, u, \underset{1}{u}, \ldots, \underset{n}{u})=0
$$

is transformed into the new equation

$$
\Phi(y, v, \underset{1}{v}, \ldots, \underset{m}{v})=0
$$

of the order $m=n+k$. Let us present the obtained equation in the form of equality

$$
F_{1}(x, u, \underset{1}{u}, \ldots, \underset{n}{u})=\lambda F_{2}(y, v, \underset{1}{v}, \ldots, \underset{s}{v})=0 .
$$

Here $\lambda$ is the differential operator of the order $r: r+s=n+k$ (so it is possible to carry out factorization of the equation $\Phi(y, v, \underset{1}{v}, \ldots, \underset{m}{v})=0$ by means of $\left.F_{2}\left(y, v, v_{1}, \ldots, v\right)=0\right)$. In this case we say that the equations

$$
F_{1}(x, u, \underset{1}{u}, \ldots, \underset{n}{u})=0 \quad \text { and } \quad F_{2}(y, v, \underset{1}{v}, \ldots, \underset{s}{v})=0
$$

are connected by the nonlocal transformation $\tau$.

If invariance algebras of these equations have different dimensions, we can put "extra" symmetries of one equation into correspondence with nonlocal symmetry of another equation. We use nonlocal transformations of variables $\tau$ for construction of formulae for generation of solutions both in the case of nonlocal invariance of the equation

$$
F_{1}(x, u, \underset{1}{u}, \ldots, \underset{n}{u})=F_{1}(y, v, \underset{1}{v}, \ldots, \underset{n}{v})=0
$$

and for other equations. If the equation

$$
F_{2}\left(y, v, \underset{1}{v}, \ldots, v_{s}\right)=0
$$


is linear and homogeneous, the transformation $\tau$ allows construction of nonlinear nonlocal superposition formulae for solutions of the equation

$$
F_{1}(x, u, \underset{1}{u}, \ldots, \underset{n}{u})=0 .
$$

The approach to investigation of nonlocal symmetries of DEs described above allows treating them as a basis for an algorithm enabling construction of new solutions from one or more given solutions.

In the following section we discuss in more detail application of nonlocal symmetries to construction of explicit superposition formulae.

\section{On symmetries and superposition principles of nonlinear heat equations}

Nonlinear equations of the class

$$
u_{t}-\partial_{x}\left[h(u)+g(u) u_{x}\right]=f(x, u)
$$

were studied in a number of papers, e.g. [12, 14, 25, 26, 27, 28].

Nonclassical (conditional) symmetries of nonlinear heat equations of the general form

$$
u_{t}=u_{x x}+f(u)
$$

were completely described in [14]. In [12] nonclassical partial symmetries of equations from the family

$$
u_{t}=\left(g(u) u_{x}\right)_{x}+f(u)
$$

are constructed. These symmetries are actually conditional symmetries of the considered equations $[6,7,23]$.

Nonlocal symmetries of some equations from the class

$$
u_{t}-\partial_{x}\left[\phi\left(u, u_{x}\right)\right]=0
$$

were found by V.V. Pukhnachev [25, 26] (in particular, the conditions on the function $\phi$, under which the transformation maps the equation into itself). The initial equation can be transformed by the Lagrange transformation

$$
\xi=\int_{0}^{x} u(y, t) d y+\int_{0}^{t} \phi\left(u(0, s), u_{x}(0, s)\right) d s, \quad \omega(\xi, t)=[u(x, t)]^{-1}
$$

into an equation from the same class

$$
\omega_{t}=\left[-\omega \phi\left(\omega^{-1},-\omega^{-3} \omega_{\xi}\right)\right]_{\xi} .
$$

Further we will review different approaches to superposition principles for solutions of nonlinear differential equations.

One of examples of superposition principles for nonlinear differential equations is given by the Bianchi permutability theorem for the sine-Gordon equation, which is adduced e.g. in [32]. Permutability of auto-Bäcklund transformations with different parameters allows constructing of an infinite family of soliton-like solutions for the sine-Gordon equation by means of extension of two one-soliton solutions which correspond to different values of parameters. The same method was applied by H.D. Wahlquist and F.B. Estabrook for the Korteveg-de Vries equation [33]. 
The idea proposed by S.E. Johnes and W.F. Ames [30] was developed in [29]. Two solutions $\stackrel{(1)}{u}$ and $\stackrel{(2)}{u}$ of the nonlinear equation

$$
F(x, u, u, \ldots, u)=0
$$

may be used by means of a procedure

$$
\Lambda(\stackrel{1}{u}, \stackrel{(2)}{u})=\stackrel{(1)}{u} * \stackrel{(2)}{u}
$$

to construct a new solution $\stackrel{(3)}{u}$ of the same equation:

$$
\Lambda: \quad(\stackrel{(1)}{u}, \stackrel{(2)}{u}) \rightarrow \stackrel{(3)}{u} .
$$

(Here $*$ denotes a procedure combining these two solutions into a new one.)

It was noted in [29] that a superposition principle is a symmetry of the system of two copies of the same equation

$$
F(x, \stackrel{(1)}{u}, \stackrel{(1)}{u}, \ldots, \stackrel{(1)}{u})=0, \quad F(x, \stackrel{(2)}{u}, \underset{1}{u}, \ldots, \stackrel{(2)}{u})=0 .
$$

They looked for symmetry operators of system (2) of the form

$$
\Gamma=\eta(\stackrel{(1)}{u}, \stackrel{(2)}{u}) \partial_{(\underset{u}{u}}
$$

whereas the representation symmetry operators of a single equation in the classical approach are

$$
\Gamma=\eta(x, \stackrel{(1)}{u}) \partial_{(\underset{u}{(1)}} .
$$

The corresponding algorithm for generation of solutions is implemented by means of calculating of the one-parameter invariance group of system (2)

$$
\stackrel{(3)}{u}=\stackrel{(1)}{u},=f(\stackrel{(1)}{u}, \stackrel{(2)}{u} ; a)
$$

associated with the operator (3). Here $a$ is the group parameter. The algorithm actually represents a realization of the solution superposition principle for the equation under consideration.

The nonlinear heat equations of the class

$$
u_{0}+\partial_{1}\left(C_{1}(u)+C_{2}(u) u_{1}\right)=0
$$

remained an object of interest for many authors [2, 3, 14] for a long time. In particular, this class of equations (4) includes the Burgers equation

$$
u_{0}+u u_{1}-u_{11}=0
$$

and the nonlinear heat equation

$$
u_{0}-\partial_{1}\left(u^{-2} u_{1}\right)=0,
$$

which admit linearization by nonlocal transformation of variables. It is well known that the equation (5) is connected with linear heat equation

$$
v_{0}-v_{11}=0
$$


by the Cole-Hopf substitution

$$
u=-2(\ln v)_{1} .
$$

The nonlocal transformation which provides linearization of equation (6) was first found in [39] and then re-discovered in [40]. It can be presented in the form [8]

$$
u\left(x_{0}, x_{1}\right)=\frac{1}{v_{1}\left(y_{0}, y_{1}\right)}, \quad x_{1}=v\left(y_{0}, y_{1}\right), \quad x_{0}=y_{0} .
$$

Here $x=\left(x_{0}, x_{1}\right)$ and $y=\left(y_{0}, y_{1}\right)$ are tuples of old and new independent variables correspondingly. This fact was utilized for constructing of the nonlocal generating formulae and formulae of nonlinear superposition principle for equation (6) in [8] and for (5) in this paper. These generating formulae are constructed with symmetry operators of the linear heat equation (7).

The invariance algebra of the linear heat equation (7) was first calculated by S. Lie and is adduced in the standard texts e.g. by Ovsiannikov [37] and Olver [18]. A basis of this algebra consists of the operators:

$$
\begin{aligned}
& P_{0}=\partial_{0}, \quad P_{1}=\partial_{1}, \quad I=v \partial_{v}, \quad D=2 y_{0} \partial_{0}+y_{1} \partial_{1}, \\
& G=y_{0} \partial_{1}-\frac{1}{2} y_{1} v \partial_{v}, \quad F=y_{0}^{2} \partial_{0}-y_{0} y_{1}-\frac{1}{4}\left(y_{1}^{2}+2 y_{0}\right) v \partial_{v}, \quad S_{b}=b\left(y_{0}, y_{1}\right) \partial_{v},
\end{aligned}
$$

where $b=b\left(y_{0}, y_{1}\right)$ is an arbitrary solution of $(7)$, i.e. $b_{0}=b_{11}$.

The operators $P_{1}$ and $G$ were applied in [8] to construction of generating formulae for equation (5). The operators $P_{0}$ and $P_{1}$ were used for the same purposes for equation (6).

In the present paper new formulae for generating of solutions for equations (5) and (6) were found. We also constructed new solutions of equation (5) and (6) with the nonlocal superposition formulae

$$
\begin{aligned}
& \stackrel{(3)}{u}\left(x_{0}, x_{1}\right)=-2 \partial_{1} \ln (\stackrel{(1)}{\tau}+\stackrel{(2)}{\tau}), \quad-2 \partial_{1} \ln (\stackrel{(k)}{\tau})=\stackrel{(k)}{u}, \\
& -2 \partial_{0} \ln \stackrel{(k)}{\tau}=\stackrel{(k)}{u}-\frac{1}{2}(\stackrel{(k)}{u})^{2}, k=1,2 .
\end{aligned}
$$

for the Burgers equation and [8]

$$
\begin{aligned}
& \stackrel{(3)}{u}\left(x_{0}, x_{1}\right)=\stackrel{(1)}{u^{-1}}\left(x_{0}, \stackrel{(1)}{\tau}\right)+u^{-1}\left(x_{0}, \stackrel{(2)}{\tau}\right), \quad \stackrel{(1)}{u}\left(x_{0}, \stackrel{(1)}{\tau}\right) d \stackrel{(1)}{\tau}=\stackrel{(2)}{u}\left(x_{0}, \stackrel{(2)}{\tau}\right) d \stackrel{(2)}{\tau}, \\
& x_{1}=\stackrel{(1)}{\tau}+\stackrel{(2)}{\tau}, \quad \stackrel{(k)}{\tau_{0}}=\tau_{1}{ }^{-2} \stackrel{(k)}{\tau_{11}} u^{-2}\left(x_{0}, \stackrel{(k)}{\tau}\right), \quad k=1,2 .
\end{aligned}
$$

for equation (6) . Here $\tau$ 's denote the functional parameters to be excluded from these formulae.

\section{Formulae for generating of solutions}

We can construct new formulae for generating of solutions of linearizable equations by means of combining symmetry operator formulae for generating of solutions of linear equations and linearizing transformations.

Namely, let $L$ be a linear differential equation, $F$ a nonlinear differential equation and $\tau$ a nonlocal transformation which linearizes the equation $F$ to the equation $L$. If $L$ admits a Lie symmetry operator $X$ being a vector field on the space of independent and dependent variables then the corresponding differential operator $Q$ acting in the space of unknown functions of $L$ maps any solution $\stackrel{(1)}{v}$ of $L$ to the other solution

$$
\stackrel{(2)}{v}=Q \stackrel{(1)}{v} \text {. }
$$


Then the nonlocal mapping $\tau$ allows us to obtain a new solution $\stackrel{(2)}{u}=\tau^{-1} \stackrel{(2)}{v}$ of $F$ from its known solution $\stackrel{(1)}{u}=\tau^{-1} \stackrel{(1)}{v}$. Here $\tau^{-1}$ is treated in a certain way. Therefore, in the case of direct utilization of the transformation $\tau$ for generating of new solutions of $F$ we have to make several steps $\stackrel{(1)}{u} \rightarrow \stackrel{(1)}{v} \rightarrow \stackrel{(2)}{v} \rightarrow \stackrel{(2)}{u}$. At the same time, it is possible to derive a formula for generating of new solutions of $F$ without involving solutions of linear equations. This way is preferable since it allows to avoid cumbersome calculations and to produce solutions in one step.

Thus, combining the Cole-Hopf substitution linearizing the Burgers equation (5) to the linear heat equation (7) and the action of the Lie symmetry operator $P_{0}=\partial_{0}$ on solutions of (7), we obtained the following result.

Theorem 1. The generating formula for equation (5), which is associated with the Lie symmetry operator $P_{0}$ of (7), has the form

$$
\stackrel{(2)}{u}=\stackrel{(1)}{u}+\frac{\stackrel{(1)}{u_{0}}}{-\frac{1}{2} u_{1}^{(1)}+\frac{1}{4}(\stackrel{1)}{u})^{2}} .
$$

Applying the formula (12) iteratively, we construct chains of solutions of the Burgers equation (5):

$$
\begin{aligned}
& \text { 1. } \frac{2}{e^{-x_{0}-x_{1}-1}} \rightarrow-2 \rightarrow \cdots \rightarrow-2 \rightarrow \cdots \\
& \text { 2. }-2\left[1+\frac{1}{x_{1}+2 x_{0}+k}\right] \rightarrow-2\left[1+\frac{1}{x_{1}+2 x_{0}+k+2}\right] \\
& \quad \rightarrow-2\left[1+\frac{1}{x_{1}+2 x_{0}+k+4}\right] \rightarrow \cdots
\end{aligned}
$$

here $k$ is an arbitrary constant;

$$
\begin{aligned}
& \text { 3. } \begin{array}{l}
x_{1} \\
x_{0}
\end{array} \quad \frac{x_{1}\left(6 x_{0}-x_{1}^{2}\right)}{x_{0}\left(2 x_{0}-x_{1}^{2}\right)} \rightarrow \frac{x_{1}\left(60 x_{0}^{2}-20 x_{0} x_{1}^{2}+x_{1}^{4}\right)}{x_{0}\left(12 x_{0}^{2}-12 x_{0} x_{1}^{2}+x_{1}^{4}\right)} \\
& \quad \rightarrow \frac{x_{1}\left(840 x_{0}^{3}-420 x_{0}^{2} x_{1}^{2}+42 x_{0} x_{1}^{4}-x_{1}^{6}\right)}{x_{0}\left(120 x_{0}^{3}-180 x_{0}^{2} x_{1}^{2}+30 x_{0} x_{1}^{4}-x_{1}^{6}\right)} \rightarrow \cdots ; \\
& \text { 4. } \quad-1-2 \tanh \left(x_{0}+x_{1}\right) \rightarrow-\frac{13+14 \tanh \left(x_{0}+x_{1}\right)}{5+4 \tanh \left(x_{0}+x_{1}\right)} \rightarrow-\frac{121+122 \tanh \left(x_{0}+x_{1}\right)}{41+40 \tanh \left(x_{0}+x_{1}\right)} \rightarrow \cdots .
\end{aligned}
$$

Consider the Lie symmetry operator $P_{0}+P_{1}=\partial_{0}+\partial_{1}$ of $(7)$ and the corresponding operator formulae for generating of solutions of the linear heat equation

$$
\stackrel{(2)}{v}=\left(\partial_{0}+\partial_{1}\right) \stackrel{(1)}{v} \text {. }
$$

Theorem 2. The generating formula for equation (5), which is associated with the Lie symmetry operator $P_{0}+P_{1}$ of $(7)$, has the form

$$
\stackrel{(2)}{u}=\stackrel{(1)}{u}-2 \frac{\stackrel{(1)}{u_{0}}+\stackrel{(1)}{u_{1}}}{\left(\mathfrak{u}_{1}\right)-\frac{1}{2}(\stackrel{(1)}{u})^{2}+\stackrel{(1)}{u}} .
$$

Similarly to the previous case, chains of solutions of equation (5) can be obtained by formula (13):

1. $C \rightarrow C \rightarrow \cdots$; 


$$
\begin{aligned}
& \text { 2. } \frac{2}{e^{-x_{0}-x_{1}}-1} \rightarrow-2 \rightarrow-2 \rightarrow \cdots ; \\
& \text { 3. }-2\left[1+\frac{1}{x_{1}+2 x_{0}+k}\right] \rightarrow-2\left[1+\frac{2}{2 x_{1}+4 x_{0}+2 k+3}\right] \rightarrow-2\left[1+\frac{1}{x_{1}+2 x_{0}+k+3}\right] \\
& \quad \rightarrow-2\left[1+\frac{2}{2 x_{1}+4 x_{0}+2 k+9}\right] \rightarrow \cdots ; \\
& \text { 4. } \frac{x_{1}}{x_{0}} \rightarrow-\frac{-6 x_{0} x_{1}+x_{1}^{3}-2 x_{1}^{2} x_{0}+4 x_{0}}{x_{0}\left(2 x_{0}-x_{1}^{2}+2 x_{1} x_{0}\right)} \\
& \quad \rightarrow \frac{24 x_{1} x_{0}^{3}+48 x_{0}^{3}-4 x_{1}^{3} x_{0}^{2}-48 x_{1}^{2} x_{0}^{2}-60 x_{1} x_{0}^{2}+4 x_{1}^{4} x_{0}+20 x_{1}^{3} x_{0}-x_{1}^{5}}{x_{0}\left(8 x_{0}^{3}-4 x_{1}^{2} x_{0}^{2}-24 x_{1} x_{0}^{2}-12 x_{0}^{2}+4 x_{1}^{3} x_{0}+12 x_{1}^{2} x_{0}-x_{1}^{4}\right)} \rightarrow \cdots \\
& \text { 5. } \quad-1-2 \tanh \left(x_{0}+x_{1}\right) \rightarrow-\frac{23+22 \tanh \left(x_{0}+x_{1}\right)}{7+8 \tanh \left(x_{0}+x_{1}\right)} \rightarrow-\frac{337+338 \tanh \left(x_{0}+x_{1}\right)}{113+112 \tanh \left(x_{0}+x_{1}\right)} \rightarrow \cdots .
\end{aligned}
$$

We also combine action of the Lie symmetry operator $P_{0}+P_{1}$ on solutions of (7) with the linearizing transformation of equation (6) to the linear heat equation (7).

Theorem 3. The generating formula for equation (6), which is associated with the Lie symmetry operator $P_{0}+P_{1}$ of $(7)$, has the form

$$
\begin{aligned}
& \stackrel{(2)}{u}\left(x_{0}, x_{1}\right)=(\stackrel{(1)}{u})^{5}\left[\left(\stackrel{(1)}{u_{\tau}}\right)^{2}-\stackrel{(1)}{u_{0}}(\stackrel{(1)}{u})^{3}-\stackrel{(1)}{u_{\tau}}(\stackrel{(1)}{u})^{2}\right]^{-1}, \\
& x_{1}=-\stackrel{(1)}{u}_{\tau}(\stackrel{(1)}{u})^{-3}+(\stackrel{(1)}{u})^{-1} .
\end{aligned}
$$

After applying formula (14) to the stationary solution

$$
\stackrel{(1)}{u}\left(x_{0}, x_{1}\right)=\frac{-1}{C_{1} x_{1}+C_{2}}
$$

of equation (6), we obtain the solution

$$
\stackrel{(2)}{u}\left(x_{0}, x_{1}\right)=\frac{-1}{C_{1} x_{1}} .
$$

Therefore, this solution is fixed under action of (14).

The next chosen operator from the Lie algebra (8) is $D=2 y_{0} \partial_{0}+y_{1} \partial_{1}$. It acts on solutions

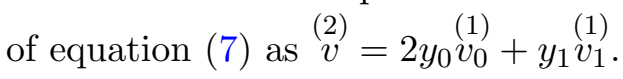

Theorem 4. The generating formula for equation (6), which is associated with the Lie symmetry operator $D$ of (5), has the form

$$
\stackrel{(2)}{u}=\stackrel{(1)}{u}+\frac{2 x_{0} \stackrel{(1)}{u_{0}}+\stackrel{(1)}{u}+x_{1} \stackrel{(1)}{u_{1}}}{-x_{0}\left(\stackrel{(1)}{u_{1}}-\frac{1}{2}(\stackrel{(1)}{u})^{2}\right)-\frac{x_{1}}{2} \stackrel{(1)}{u}} .
$$

The following chains of solutions of equation (5) are obtained with the formula (15):

1. $C \rightarrow C+\frac{1}{\frac{C}{2} x_{0}-\frac{1}{2} x_{1}} \rightarrow C+\frac{4 C^{2} x_{0}-4 C x_{1}+4}{C^{3} x_{0}^{2}-2 C^{2} x_{0} x_{1}+4 C x_{0}+C x_{1}^{2}-2 x_{1}} \rightarrow \cdots$;

2. $\frac{2}{e^{-x_{0}-x_{1}}-1} \rightarrow-2\left[1+\frac{1}{x_{1}+2 x_{0}}\right] \rightarrow-2\left[1+\frac{4 x_{0}+2 x_{1}+1}{4 x_{0}^{2}+4 x_{0}+4 x_{0} x_{1}+x_{1}^{2}+x_{1}}\right] \rightarrow \cdots$;

3. $\frac{x_{1}}{x_{0}} \rightarrow \frac{x_{1}}{x_{0}} \rightarrow \cdots ;$ 
4. $-1-2 \tanh \left(x_{0}+x_{1}\right) \rightarrow-\frac{13 x_{0}+5 x_{1}+2+\left(14 x_{0}+4 x_{1}+4\right) \tanh \left(x_{0}+x_{1}\right)}{5 x_{0}+x_{1}+\left(4 x_{0}+2 x_{1}\right) \tanh \left(x_{0}+x_{1}\right)} \rightarrow \cdots$.

Now we construct new solutions of the equation (5) by application of the superposition principle (9).

1. For the solutions $\stackrel{(1)}{u}=\frac{x_{1}}{x_{0}}$ and $\stackrel{(2)}{u}=1+\frac{2}{x_{0}-x_{1}}$ we have

$$
\stackrel{(3)}{u}=\frac{C_{1} x_{1} e^{-\frac{x_{1}^{2}}{4 x_{0}}}-C_{2} e^{\frac{x_{0}}{4}-\frac{x_{1}}{2}}\left(x_{0}^{\frac{5}{2}}-x_{0}^{\frac{3}{2}} x_{1}+2 x_{0}^{\frac{3}{2}}\right)}{x_{0}\left(C_{1} e^{-\frac{x_{1}^{2}}{4 x_{0}}}-C_{2} e^{\frac{x_{0}}{4}-\frac{x_{1}}{2}}\left(x_{0}^{\frac{3}{2}}-x_{0}^{\frac{1}{2}} x_{1}\right)\right)} .
$$

2. If $\stackrel{(1)}{u}=\frac{x_{1}}{x_{0}}, \stackrel{(2)}{u}=-1-2 \tanh \left(x_{0}+x_{1}\right)$ then

$$
\stackrel{(3)}{u}=-\frac{C_{1} x_{1} e^{-\frac{x_{1}^{2}}{4 x_{0}}}+i C_{2} e^{\frac{5 x_{0}}{4}+\frac{x_{1}}{2}} x_{0}^{\frac{3}{2}}\left(2 \sinh \left(x_{0}+x_{1}\right)+\cosh \left(x_{0}+x_{1}\right)\right)}{x_{0}\left(-C_{1} e^{-\frac{x_{1}^{2}}{4 x_{0}}}+i C_{2} \cosh \left(x_{0}+x_{1}\right) e^{\frac{5 x_{0}}{4}+\frac{x_{1}}{2}} x_{0}^{\frac{1}{2}}\right)} .
$$

3. The solutions $\stackrel{(1)}{u}=\frac{x_{1}}{x_{0}}, \stackrel{(2)}{u}=\frac{2}{e^{-x_{0}-x_{1}}}$ generate the solution

$$
\stackrel{(3)}{u}=\frac{C_{1} x_{1} e^{-\frac{x_{1}^{2}}{4 x_{0}}}-2 C_{2} e^{x_{0}+x_{1}} x_{0}^{\frac{3}{2}}}{x_{0}\left(C_{1} e^{-\frac{x_{1}^{2}}{4 x_{0}}}-C_{2} x_{0}^{\frac{1}{2}}+C_{2} x_{0}^{\frac{1}{2}} e^{x_{0}+x_{1}}\right)} .
$$

In a similar way, we construct new solutions of equation (6) with superposition principle (10). Thus, from the stationary solutions $\stackrel{(1)}{u}=-\frac{1}{x_{1}}$ and $\stackrel{(2)}{u}=-\frac{1}{2 x_{1}}$ we obtain the solution

$$
\stackrel{(3)}{u}=\frac{2 C_{1} e^{2 x_{0}}}{-1-4 C_{1} x_{1} e^{2 x_{0}}+\sqrt{1+4 C_{1} x_{1} e^{2 x_{0}}}} .
$$

With other two stationary solutions $\stackrel{(1)}{u}=\frac{1}{x_{1}}, \stackrel{(2)}{u}=-\frac{1}{x_{1}+C}$ we get

$$
\stackrel{(3)}{u}=\left[-x_{1}+2 e^{\left(\text {LambertW }\left(-\frac{1}{2}\left(x_{1}+C\right) e^{x_{0}-\frac{C_{1}}{2}}\right)-x_{0}+\frac{C_{1}}{2}\right)}-C\right]^{-1} .
$$

Note that the constructed solutions are not stationary.

All obtained solutions can be extended to parametric families of solutions with Lie symmetry transformations or by using other known formulae of generating of solutions. For instance, for any real value $r$ and any solution $v$ of the Burgers equation (5)

$$
\stackrel{(2)}{v}=-2 \frac{\stackrel{(1)}{v}}{(1)}
$$

is a solution of the same Burgers equation.

\section{Classical and nonlocal symmetries of equation $\operatorname{Slid}\left(u_{\mu \nu}\right)=0$}

Consider the equation

$$
u_{11} u_{22}-u_{12}^{2}-\left(u_{00} u_{22}-u_{02}^{2}\right)-\left(u_{00} u_{11}-u_{01}^{2}\right)=0 .
$$


It is an essentially nonlinear second order scalar PDE in three independent variables, which can be linearized to the $(1+2)$-dimensional d'Alembert equation. Its left hand side is the sum of algebraic complements to the diagonal elements of the adjoint matrix to the Hesse matrix $\left(u_{\mu \nu}\right)$ with the metric tensor $\left(g_{\mu \nu}\right)=\operatorname{diag}(1,-1,-1)$ of the Minkowski space $\mathbb{R}^{1,2}$. This equation was first investigated in [34]. One of the authors of [34] (W.I. Fushchych) suggested the notation

$$
\operatorname{Slid}\left(u_{\mu \nu}\right):=g_{\mu \nu} A^{\mu \nu}=0 .
$$

Here $A^{\mu \nu}$ is the algebraic complement to the element $u_{\mu \nu}$ of the Hessian matrix. Hereafter the indices $\mu$ and $\nu$ run from 0 to 2 , and we assume summation over repeated indices. To the best of our knowledge, the Lie symmetries of equation (16) had not been studied. We search for the maximal Lie invariance algebra $\mathcal{A}$ of equation (16) by the classical technique $[18,37]$.

In view of the infinitesimal invariance criterion, any operator

$$
X=\xi^{\mu}(x, u) \partial_{\mu}+\eta(x, u) \partial_{u}, \quad x=\left(x_{0}, x_{1}, x_{2}\right),
$$

from $\mathcal{A}$ satisfies the equation

$$
\left.X^{(2)}\left(\operatorname{Slid}\left(u_{\mu \nu}\right)\right)\right|_{M}=0
$$

where $X^{(2)}$ is the standard second-order prolongation of $X, M$ is the manifold determined by equation (16) in the second-order jet space. This is a necessary and sufficient condition for infinitesimal invariance of equation (16). We confine to the manifold $M$ e.g. with the equality

$$
u_{22}=\frac{u_{00} u_{11}-u_{01}^{2}-u_{02}^{2}+u_{12}^{2}}{u_{11}-u_{00}} .
$$

and then split the obtained expression with respect to the unconstrained derivatives $u_{\mu \nu},(\mu, \nu) \neq$ $(2,2)$, and $u_{\mu}$. After solving the derived system of determining equations, we find the general form of coefficients of operators from $\mathcal{A}$ :

$$
\begin{aligned}
& \xi^{0}=C_{1} x_{0}+C_{2} x_{1}+C_{3} x_{2}+C_{4}, \\
& \xi^{1}=C_{2} x_{0}+C_{1} x_{1}-C_{5} x_{2}+C_{6}, \\
& \xi^{2}=C_{3} x_{0}+C_{5} x_{1}+C_{1} x_{2}+C_{7}, \\
& \eta=C_{8} x_{0}+C_{9} x_{1}+C_{10} x_{2}+C_{11} u+C_{12} .
\end{aligned}
$$

Therefore, the maximal Lie invariance algebra $\mathcal{A}$ of equation (16) is 12-dimensional. A basis of $\mathcal{A}$ consists of the operators

$$
\begin{aligned}
& P_{0}=\partial_{0}, \quad P_{1}=\partial_{1}, \quad P_{2}=\partial_{2}, \quad P_{3}=\partial_{u}, \quad I=u \partial_{u}, \\
& J_{01}=x_{0} \partial_{1}+x_{1} \partial_{0}, \quad J_{02}=x_{0} \partial_{2}+x_{2} \partial_{0}, \quad J_{12}=-x_{2} \partial_{1}+x_{1} \partial_{2}, \\
& D=x_{0} \partial_{0}+x_{1} \partial_{1}+x_{2} \partial_{2}, \quad Q_{0}=x_{0} \partial_{u}, \quad Q_{1}=x_{1} \partial_{u}, \quad Q_{2}=x_{2} \partial_{u} .
\end{aligned}
$$

The algebra $\mathcal{A}$ can be used for finding of exact solutions of equation (16). Exhaustive investigation of Lie reductions of equation (16) includes the following steps: 1) construction of optimal systems of one- and two-dimensional subalgebras of the algebra $\mathcal{A}$ and corresponding sets of ansatzes of codimensions one and two; 2) reduction of the initial equations with these ansatzes to partial differential equations in two independent variables or ordinary differential equations; 3) solving of the reduced equations.

Here we restrict ourselves to particular reductions with respect to one-dimensional subalgebras. Below we list basis operators of these subalgebras, corresponding ansatzes, reduced 
equations for the new unknown function $\varphi$ in two independent variables and some exact solutions of the initial equation, which are found in the following way.

1) $P_{1}+Q_{0}$ :

$$
\begin{aligned}
& u=x_{0} x_{1}+\varphi\left(x_{0}, x_{2}\right), \quad \varphi_{02}^{2}-\varphi_{00} \varphi_{22}+1=0 ; \\
& u=x_{0} x_{1}+\frac{1}{2} x_{2}^{2}+\frac{1}{2} x_{0}^{2}+C_{1} x_{0}+C_{2} .
\end{aligned}
$$

2) $P_{1}+Q_{1}$ :

$$
\begin{aligned}
u= & \frac{1}{2} x_{1}^{2}+\varphi\left(x_{0}, x_{2}\right), \quad \varphi_{02}^{2}-\varphi_{00} \varphi_{22}+\varphi_{22}-\varphi_{00}=0 ; \\
u= & \frac{1}{2} x_{1}^{2} \mp \frac{1}{2} \sqrt{\left(x_{2}^{2}-x_{0}^{2}\right)\left(x_{2}^{2}-x_{0}^{2}+4 C_{1}\right)} \\
& \mp C_{1} \ln \left(2 C_{1}+x_{2}^{2}-x_{0}^{2}+\sqrt{\left(x_{2}^{2}-x_{0}^{2}\right)\left(x_{2}^{2}-x_{0}^{2}+4 C_{1}\right)}\right)-\frac{1}{2}\left(x_{2}^{2}-x_{0}^{2}\right)+C_{2} .
\end{aligned}
$$

3) $J_{02}$ :

$$
\begin{aligned}
u= & \varphi\left(\omega, x_{1}\right), \quad \omega=x_{0}^{2}-x_{2}^{2}, \quad \omega\left(\varphi_{\omega 1}^{2}-\varphi_{\omega \omega} \varphi_{11}\right)+2 \omega \varphi_{\omega} \varphi_{\omega \omega}-\varphi_{\omega} \varphi_{11}+\varphi_{\omega}^{2}=0 ; \\
u= & -\frac{1}{2} x_{1}^{2} \pm \frac{1}{2} \sqrt{\left(x_{0}^{2}-x_{2}^{2}\right)\left(x_{0}^{2}-x_{2}^{2}+4 C_{1}\right)} \\
& \pm C_{1} \ln \left(2 C_{1}+x_{0}^{2}-x_{2}^{2}+\sqrt{\left(x_{0}^{2}-x_{2}^{2}\right)\left(x_{0}^{2}-x_{2}^{2}+4 C_{1}\right)}\right)-\frac{1}{2}\left(x_{0}^{2}-x_{2}^{2}\right)+C_{2} .
\end{aligned}
$$

4) $J_{02}+J_{12}$ :

$$
\begin{aligned}
& u=\varphi\left(\omega_{1}, \omega_{2}\right), \quad \omega_{1}=x_{0}+x_{1}, \quad \omega_{2}=x_{2}^{2}+x_{1}^{2}-x_{0}^{2}, \\
& \omega_{1}^{2}\left(\varphi_{12}^{2}-\varphi_{11} \varphi_{22}\right)+4 \omega_{2} \varphi_{2} \varphi_{22}+4 \omega_{1} \varphi_{2} \varphi_{12}+3 \varphi_{2}^{2}=0 ; \\
& u=C_{1} \frac{\sqrt{x_{2}^{2}+x_{1}^{2}-x_{0}^{2}}}{x_{0}+x_{1}}, \quad u=\frac{C_{2}\left(x_{2}^{2}+x_{1}^{2}-x_{0}^{2}\right)+C_{1}}{x_{0}+x_{1}} .
\end{aligned}
$$

Another way of application of Lie symmetries to construction of exact solutions is generation of new solutions from known ones by symmetry transformations. We can reconstruct the Lie symmetry group $\mathcal{G}$ of equation (16) from the algebra $\mathcal{A}$ via solving of a set of Cauchy problems. As a result, we obtained that the group $\mathcal{G}$ is generated by the following transformations:

translations with respect to $x$ and $u: x_{\mu}^{\prime}=x_{\mu}+a_{\mu}, u^{\prime}=u+a_{3}$,

scale transformations with respect to $x$ and $u$ : $x_{\mu}^{\prime}=e^{a_{4}} x_{\mu}, u^{\prime}=e^{a_{5}} u$,

addition of a linear in $x$ term to $u: \quad x_{\mu}^{\prime}=x_{\mu}, u^{\prime}=u+b_{\mu} x_{\mu}$,

rotations in the plane $\left(x_{1}, x_{2}\right)$ :

$$
x_{0}^{\prime}=x_{0}, x_{1}^{\prime}=x_{1} \cos c_{1}-x_{2} \sin c_{1}, x_{2}^{\prime}=x_{1} \sin c_{1}+x_{2} \cos c_{1}, u^{\prime}=u,
$$

Lorentz rotations in the plane $\left(x_{0}, x_{1}\right)$ :

$$
x_{0}^{\prime}=x_{0} \cosh c_{2}+x_{1} \sinh c_{2}, x_{1}^{\prime}=x_{0} \sinh c_{2}+x_{1} \cosh c_{2}, x_{2}^{\prime}=x_{2}, u^{\prime}=u,
$$

Lorentz rotations in the plane $\left(x_{0}, x_{2}\right)$ :

$$
x_{0}^{\prime}=x_{0} \cosh c_{3}+x_{2} \sinh c_{3}, x_{1}^{\prime}=x_{1}, x_{2}^{\prime}=x_{0} \sinh c_{3}+x_{2} \cosh c_{3}, u^{\prime}=u,
$$

where $a_{0}, \ldots, a_{5}, b_{\mu}, c_{1}, c_{2}$ and $c_{3}$ are arbitrary constants.

Parametrical generation of solutions (18) and (19) by means of symmetry transformations gives, for example, the following new solutions:

$$
\begin{aligned}
& u=\left(x_{2} a+x_{0}\right) x_{1} \sqrt{1-a^{2}}+\frac{1}{2}\left(x_{0} a+x_{2}\right)^{2}+\frac{1}{2}\left(x_{2} a+x_{0}\right)^{2}+C_{1}\left(x_{2} a+x_{0}\right)+C_{2}, \\
& u=\frac{C_{2}\left(x_{2}^{2}+x_{1}^{2}-x_{0}^{2}\right)+C_{1}}{x_{0} \cosh c_{3}+x_{2} \sinh c_{3}+x_{1}} .
\end{aligned}
$$

Obtained solutions with parameters can be used in construction of further new solutions, for instance, by application of the nonlinear superposition principle. 


\section{Nonlocal linearization and the formula of superposition of solutions for equation (16)}

As established in [34], the $(1+2)$-dimensional nonlinear equation $\operatorname{Slid}\left(u_{\mu \nu}\right)=0$ can be reduced to a linear one by the Legendre transformation in the space of variables $x=\left(x_{0}, x_{1}, x_{2}\right)$ :

$$
\begin{aligned}
& u\left(x_{0}, x_{1}, x_{2}\right)=y_{0} v_{0}+y_{1} v_{1}+y_{2} v_{2}-v, \\
& x_{0}=v_{0}, \\
& x_{1}=v_{1}, \\
& x_{2}=v_{2},
\end{aligned}
$$

The Legendre transformation is prolonged to the first and second order derivatives in the space of variables $x$ in the following way:

$$
\begin{aligned}
& u_{0}=y_{0}, \quad u_{1}=y_{1}, \quad u_{2}=y_{2}, \\
& u_{00}=\frac{v_{11} v_{22}-v_{12}^{2}}{\delta}, \quad u_{01}=-\frac{v_{01} v_{22}-v_{20} v_{12}}{\delta}, \quad u_{11}=\frac{v_{00} v_{22}-v_{20}^{2}}{\delta}, \\
& u_{02}=\frac{v_{10} v_{21}-v_{11} v_{20}}{\delta}, \quad u_{01}=-\frac{v_{00} v_{21}-v_{20} v_{10}}{\delta}, \quad u_{22}=\frac{v_{00} v_{11}-v_{10}^{v_{12}} 2}{\delta},
\end{aligned}
$$

where $\delta=\operatorname{det}\left(v_{\mu \nu}\right) \neq 0$.

Substituting the obtained expressions for $v_{\mu \nu}$ in (16), we get the linear d'Alembert equation

$$
\square u=u_{00}-u_{11}-u_{22}=0 .
$$

Since the Legendre transformation is an involution, i.e. it coincides with its inverse transformation, application of the Legendre transformation to (21) results in the initial equation (16).

In [8] a nonlinear superposition formula was derived for any nonlinear differential equation which can be reduced by the Legendre transformation (20) to a linear homogeneous equation. In the case of equation (16) this formula has the form:

$$
\stackrel{(3)}{u}(x)=\stackrel{(1)}{u}(\tau)+\stackrel{(2)}{u}(\theta), \quad \stackrel{(1)}{u}(\tau)=\stackrel{(2)}{u} \mu(\theta), \quad x=\tau+\theta,
$$

where $x=\left(x_{0}, x_{1}, x_{2}\right)$, the subscript $\mu$ of a function $u$ denotes differentiation with respect to the $\mu$-th argument of $u$. $\tau=\left(\tau_{0}, \tau_{1}, \tau_{2}\right)$ and $\theta=\left(\theta_{0}, \theta_{1}, \theta_{2}\right)$ are tuples of parameter-functions depending on $x$.

We apply formula (22) for construction of new solutions of (16) from pairs of its known solutions. Let us choose the solutions

$$
\begin{aligned}
& \stackrel{(1)}{u}=-\sqrt{-2 C_{1}\left(x_{1}^{2}-\left(x_{0}+a x_{2}\right)^{2}-2\left(x_{0}+a x_{2}\right)\right)+2 C_{1}}+C_{2}, \\
& \left.\stackrel{(2)}{u}=-2 \sqrt{-2 C_{1}\left(\left(a x_{0}+a x_{1}+x_{2}\right)^{2}+\left(a x_{1}+a x_{2}+x_{0}\right)^{2}\right.}\right)+C_{2} .
\end{aligned}
$$

They satisfy the condition $\operatorname{det}\left(u_{\mu \nu}\right) \neq 0$. Therefore, it is possible to use formula (22) for finding of a new solution of (16). We re-denote the arguments of the first and second solutions as $\tau$ and $\theta$ respectively. Replacing $\tau_{\mu}$ by the expression $x_{\mu}-\theta_{\mu}$, we obtain the solution

$$
\begin{aligned}
\stackrel{(3)}{u}= & -\sqrt{-2 C_{1}\left(x_{1}-\theta_{1}\right)^{2}+2 C_{1}\left(a\left(x_{2}-\theta_{2}\right)+x_{0}-\theta_{0}\right)^{2}-\left(a\left(x_{2}-\theta_{2}\right)+x_{0}-\theta_{0}\right)+2 C_{1}} \\
& -2 \sqrt{2 C_{1}\left(\left(a \theta_{0}+a \theta_{1}+\theta_{2}\right)^{2}+\left(a \theta_{1}+a \theta_{2}+\theta_{0}\right)^{2}\right)}+C_{2} .
\end{aligned}
$$

The functional parameters $\theta_{0}, \theta_{1}$ and $\theta_{2}$ are found from the system

$$
R_{1}\left(-\left(a\left(x_{2}-\theta_{2}\right)+x_{0}-\theta_{0}\right)-1\right)=R_{2}\left(\theta_{0} a^{2}+\theta_{1} a^{2}+2 \theta_{2} a+\theta_{1} a+\theta_{0}\right)
$$




$$
\begin{aligned}
& R_{1}\left(x_{1}-\theta_{1}\right)=R_{2}\left(\theta_{0} a^{2}+2 \theta_{1} a^{2}+\theta_{2} a^{2}+\theta_{2} a+\theta_{0} a\right) \\
& R_{1}\left(-\left(a\left(x_{2}-\theta_{2}\right)+x_{0}-\theta_{0}\right)-1\right)=R_{2}\left(\theta_{0} a^{2}+\theta_{1} a^{2}+2 \theta_{2} a+\theta_{1} a+\theta_{0}\right),
\end{aligned}
$$

where

$$
\begin{aligned}
& R_{1}=\left(-2 C_{1}\left(x_{1}-\theta_{1}\right)^{2}+2 C_{1}\left(a\left(x_{2}-\theta_{2}\right)+x_{0}-\theta_{0}\right)^{2}-\left(a\left(x_{2}-\theta_{2}\right)+x_{0}-\theta_{0}\right)+2 C_{1}\right)^{-\frac{1}{2}}, \\
& R_{2}=2\left(2 C_{1}\left(\left(a \theta_{0}+a \theta_{1}+\theta_{2}\right)^{2}+\left(a \theta_{1}+a \theta_{2}+\theta_{0}\right)^{2}\right)\right)^{\frac{1}{2}} .
\end{aligned}
$$

\section{Conclusion}

In this work we continued investigation of nonlocal symmetries of PDEs by means of nonlocal transformations of variables. Application of nonlocal symmetries to construction of explicit superposition formulae and formulae of generation of solutions was discussed. We obtained new formulae for generation of solutions for the Burgers equation and the nonlinear heat equation. New superposition principles were constructed for them and then used for obtaining of new solutions. The formula of nonlinear superposition of solutions for the multidimensional equation

$$
\operatorname{Slid}\left(u_{\mu \nu}\right)=u_{11} u_{22}-u_{12}^{2}-\left(u_{00} u_{22}-u_{02}^{2}\right)-\left(u_{00} u_{11}-u_{01}^{2}\right)=0
$$

was applied to construction of a new solution from two known ones. Such algorithms of generating of new solutions represent new nonlocal symmetries of nonlinear equations under investigation.

All obtained solutions can be extended to parametric families by means of Lie symmetry transformations or by using other formulae for generation of solutions.

\section{Acknowledgments}

The authors would like to thank the referees for helpful suggestions and comments.

\section{References}

[1] Anco S.C., Bluman G., Derivation of conservation laws from nonlocal symmetries of differential equations, J. Math. Phys. 37 (1996), 2361-2375.

[2] Moitsheki R.J., Broadbridge P., Edwards M.P., Systematic construction of hidden nonlocal symmetries for the inhomogeneous nonlinear diffusion equation, J. Phys. A: Math. Gen. 37 (2004), 8279-8286.

[3] Galas F., New nonlocal symmetries with pseudopotentials, J. Phys. A: Math. Gen. 25 (1992), L981-L986.

[4] Papachristou C.J., Harrison K., Nonlocal symmetries and Bäcklund transformations for the self-dual YangMills system, J. Math. Phys. 29 (1988), 238-243.

[5] Ibragimov N.H. (Editor), CRC Handbook of Lie group analysis of differential equations. Vol. 1. Symmetries, exact solutions and conservation laws, CRC Press, 1994.

[6] Olver P.J., Rosenau P., The construction of special solutions to partial differential equations, Phys. Lett. A 114 (1986), 107-112.

[7] Olver P.J., Rosenau P., Group-invariant solutions of differential equations, SIAM J. Appl. Math. 47 (1987), 263-278.

[8] Fushchych W.I., Serov M.I., Tychynin V.A., Amerov T.K., On nonlocal symmetry of nonlinear heat equation, Proc. Acad. of Sci. Ukraine (1992), no. 11, 27-33.

[9] Krasil'shchik I.S., Vinogradov A.M., Nonlocal trends in the geometry of differential equations: symmetries, conservation laws and Bäcklund transformations, Acta Appl. Math. 15 (1989), 161-209.

[10] Anderson R.L., Ibragimov N.H., Lie-Bäcklund transformations in applications, SIAM, Philadelphia, 1979.

[11] Ibragimov N.H., Transformation groups applied to mathematical physics, Reidel, Dordrecht, 1985. 
[12] Dzamay A.V., Vorob'ev E.M, Infinitesimal weak symmetries of nonlinear differential equations in two independent variables, J. Phys. A: Math. Gen. 27 (1994), 5541-5549.

[13] Gandarias M.L., Bruzon M.S., Symmetry analysis and solutions for a family of Cahn-Hillard equations, Rep. Math. Phys. 46 (2000), 89-97.

[14] Clarkson P.A., Mansfield E.L., Symmetry reduction and exact solutions of a class of nonlinear heat equations, Phys. D 70 (1993), 250-288, solv-int/9306002.

[15] Ibragimov N.H., Anderson R.L., Lie-Bäcklund tangent transformations, J. Math. Anal. Appl. 59 (1977), $145-162$.

[16] Fok V.A., Hydrogen atom and non-Euclidean geometry. Preliminary announcement, Zs. Phys. 98 (1935), $145-154$.

[17] Lowner C.A., A transformation theory of the partial differential equations of gas dynamics, Nat. Advis. Comm. Aeronat. Tech. Notes (1950), no. 2065, 56 pages.

[18] Olver P.J., Applications of Lie groups to differential equations, Springer-Verlag, New York, 1993.

[19] Guthrie G.A., Recursion operators and nonlocal symmetries, Proc. Roy. Soc. London A 446 (1994), $107-114$.

[20] Guthrie G.A., Hickman M.S., Nonlocal symmetries of the KdV equation, J. Math. Phys. 34 (1993), $193-205$.

[21] Leo M., Leo R.A., Soliani G., Tempesta P., On the relation between Lie symmetries and prolongation structures of nonlinear field equations, Progr. Theoret. Phys. 105 (2001), 77-97.

[22] Olver P.J., Sanders J., Wang J-P., Ghost symmertries, J. Nonlinear Math. Phys. 9 (2002), suppl. 1, 164-172.

[23] Fushchich W.I., Serov N.I., The conditional symmetry and reduction of the nonlinear heat conduction equation, Proc. Acad. of Sci. Ukraine, Ser. A (1990), no. 7, 24-27.

[24] Tychynin V.A., Nonlocal symmetry and generating solutions for Harry-Dym type equations, J. Phys. A: Math. Gen. 27 (1994), 2787-2797.

[25] Pukhnachev V.V., Nonlocal symmetries in nonlinear heat equations, in Energy Methods in Continuum Mechanics (Oviedo, 1994), Kluwer Acad. Publ., Dordrecht, 1996, 75-99.

[26] Pukhnachev V.V., Exact solutions of the hydrodynamic equations derived from partially invariant solutions, J. Appl. Mech. Tech. Phys. 44 (2003), 317-323.

[27] Sophocleous C., Transformation properties of variable-coefficient Burgers equation, Chaos Solitons Fractals 20 (2004), 1047-1057.

[28] Sophocleous C., Further transformation properties of generalized inhomogeneous nonlinear diffusion equations with variable coefficients, Phys. A 345 (2005), 457-471.

[29] Goard J.M., Broadbridge P., Nonlinear superposition principles obtained by Lie symmetry methods, J. Math. Anal. Appl. 214 (1997), 633-657.

[30] Jones S.E., Ames W.F., Nonlinear superposition, J. Math. Anal. Appl. 17 (1967), 484-487.

[31] Konopelchenko B.G., On the general structure of nonlinear evolution equations and their Bäcklund transformations connected with the matrix non-stationary Schrödinger spectral problem, J. Phys. A: Math. Gen. 15 (1982), 3425-3437.

[32] Rogers C., Shadwick W.F., Bäcklund transformations and their applications, Academic Press, New York, 1982.

[33] Wahlquist H.D., Estabrook F.B., Bäcklund transformation for solutions of the Korteweg-de Vries equation, Phys. Rev. Lett. 31 (1973), 1386-1390.

[34] Fushchych W.I., Tychynin V.A., Generating of solutions for nonlinear equations by the Legendre transformation, Proc. Acad. Sci. Ukraine (1992), no. 7, 20-25.

[35] Fairlie D.B., Mulvey J.A., Integrable generalizations of the two-dimensional Born-Infeld equation, J. Phys. A: Math. Gen. 27 (1994), 1317-1324.

[36] Kumei S., Bluman G.W., When nonlinear differential equations are equivalent to linear differential equations, SIAM J. Appl. Math. 42 (1982), 1157-1173.

[37] Ovsiannikov L.V., Group analysis of differential equations, Academic Press, 1982.

[38] Bluman G.W., Kumei S., Symmetries and differential equations, Springer, Berlin, 1989.

[39] Storm M.L., Heat conduction in simple metals, J. Appl. Phys. 22 (1951), 940-951.

[40] Bluman G., Kumei S., On the remarkable nonlinear diffusion equation $(\partial / \partial x)\left[a(u+b)^{-2}(\partial u / \partial x)\right]-\partial u / \partial t=0$, J. Math. Phys. 21 (1980), 1019-1023. 